\title{
Quantum Faraday Excitations in Degenerate Electron-Ion Plasma
}

\author{
M. Akbari-Moghanjoughi ${ }^{1}$ and B. Eliasson ${ }^{2}$ \\ ${ }^{1}$ Faculty of Sciences, Department of Physics, \\ Azarbaijan Shahid Madani University, 51745-406 Tabriz, Iran \\ ${ }^{2} S U P A$, Physics Department, John Anderson Building, \\ University of Strathclyde, Glasgow G4 ONG, Scotland, UK
}

(Dated: December 17, 2019)

\begin{abstract}
A hydrodynamic two-fluid model encompassing inertialess electrons of arbitrary degree of degeneracy and cold ions using the quasineutrality assumption are reduced to an effective nonlinear Schrödinger equation (NLSE) which is used to investigate driven electrostatic ion quasiparticle excitations. The quantized frequency spectrum of these quasi-particle excitations in a one-dimensional quasineutral electron-ion plasma confined in rectangular potential well is calculated. The spectrum shows a quadratic energy level increase quite similar to that of a single electron confined in a hard box, with much reduced level spacings proportional to the electron-to-ion mass ratio. The parametrically driven NLSE is also used to study the quantum Faraday excitations in both weakly and fully nonlinear regimes by employing the pseudo-potential technique. The quantization criterion for fully nonlinear driven quantum Faraday excitations in an arbitrary degenerate plasma confined in a hard box of length $l$ is derived, and it is shown that these excitations constitute a full frequency spectrum level starting with those of small amplitude, high frequency sinusoidal quasi-particles up to the topmost zero-frequency level solitary quasi-particle excitations (quasi-soliton level).

PACS numbers: 52.30.-q,71.10.Ca, 05.30.-d
\end{abstract}




\section{INTRODUCTION}

Faraday waves [1] are well-known suitably driven parametric excitations which occur on the surface of a variety of viscous fluids. These oscillations which take place in a quantized quantity of the driver frequency above a critical external force amplitude appear as spectacular symmetric patterns on the surface of liquids [2-4]. Despite numerous efforts to investigate these nonlinear patterns, many aspects of the excitations are still poorly understood, due to the complexity of the physical phenomenon. The complexity is basically due to the large number of parameters which can be varied in the system, such as the driving frequency and amplitude, the fluid viscosity and other thermodynamic state parameters, different kinds of fluid instabilities, etc. $[5,6]$. The theory, however, may as well apply to microscopic environments such as plasmas consisting multi-species atomic fluids. The problem may become more appealing when quantum effects arise due to reduced inter-particle distances. However, no such extension has been considered in the past, mainly due to the lack of theory for solid state plasmas such as metals, as a preferred platform. Surface Faraday (gravity) waves has recently been investigated in liquid metals [7] driven by frequencies in the range 20-80 $\mathrm{Hz}$, which show highly symmetric patterns. On the other hand, electrostatic ion Faraday waves may be easily excited using a lower band RF frequency drive around the ion plasma frequency $\omega_{i}=\sqrt{4 \pi e^{2} n_{0} / m_{i}}$ in which $n_{0}$ is the ion number density, $e$ the unit charge, and $m_{i}$ the ion mass. There are also recent suggestions for energy extraction by parametric Faraday wave resonance in a magnetic fluid [8].

Quantum effects play an important role in a broad range of physical phenomena involving particle-particle and particle-potential interactions. In solids and dense plasmas the nature of mutual interaction of constituent particles radically change when the inter-particle distances is lowered either by a pressure increase or temperature decrease beyond a scale-length characteristic of quantum regime where overlapping of the single-particle wave functions start to emerge [9]. However, recent studies of the quantum electron gas [10] provides a new statistical interpretation of the quantum effects, other than the conventional Copenhagen probabilistic interpretation, based on the interference between single-particle and collective motion of constituent particles in a statistical ensemble. The study of statistical quantum effects has a long scientific history of more than seven decades, and started by the pioneering works of several prominent researchers [11-17]. In recent years, the investigation of collective 
quantum excitations has received a renewed interest [18-34] due to its broad technological applications in the rapidly developing fields of nano-electronics, quantum optics, plasmonics, and quantum device fabrication [35-39].

Due to the large degree of freedom and complexity of mutual interactions in quantum plasmas, the dynamic simulation of decomposed wave functions of $N>10$ number of singleparticle Schrödinger equations is a formidable task. There are, however, other effective models such as quantum kinetic [40] and hydrodynamic [41] models which fundamentally reduce the degree of complexity of the calculations. Particularly, the quantum hydrodynamic approach has been found to provide unique and far-reaching analytic results which brings into view interesting aspects of collective interactions in dense plasmas [42]. Using hydrodynamic models to study basic plasma phenomena in quantum ionized environments such as the electromagnetic wave propagations and interactions, multistream quantum phenomena, wave instabilities and various nonlinear effects reveal even more collective aspects which are significantly distinguished from the classical counterparts.

The quantum hydrodynamic model may also be cast into a collective nonlinear Schrödinger equation (NLSE) for the investigation of electrostatic ion excitations or a collective NLSE-Poisson system for studying electron plasma oscillations in quantum plasmas [43]. The linearized Schrödinger-Poisson (SP) model has been recently used to investigate quantum features of a degenerate electron gas by means of a coupled pseudo-force system [44]. Analytical and numerical studies of the NLSE have found numerous applications in quantum mechanics as well as in the description of quasi-monochromatic wave propagation in weakly nonlinear media [45], including laser-plasma interaction, nonlinear optics, gravity waves, etc. The NLSE is closely related to the Zakharaov system [46-48] which is used to describe the propagation of high-frequency waves and their coupling to low-frequency oscillations, such as Langmuir oscillations in an ionized plasma and their interaction with ion acoustic waves, and interactions between the short- and long-wave gravitational disturbances in the atmosphere [49]. The NLSE and Zakharov systems in inhomogeneous plasmas [50-53] exhibit interesting dynamics involving localized excitations, solitons and chaos.

The aim of this paper is to use a quantum hydrodynamic model of electrons and ions to investigate quantum Faraday quasi-particle excitations in a plasma with Fermi-degenerate electrons. The paper is organized as follows. We provide the mathematical model of quantum electrostatic ion excitations in Sec. II. The quantized frequency spectrum of free electrostatic 
quasi-particle excitations of ions in the arbitrary degenerate quasineutral electron gas is presented in Sec. III. The weakly nonlinear quantum Faraday quasi-particle excitations in a parametrically driven ions in an arbitrary degenerate electron fluid is studied in Sec. IV. The fully nonlinear quantum Faraday quasi-particle excitations and their wavefunction along with the corresponding quantized frequency spectrum is investigated in Sec. V, and the damped parametrically driven excitations are presented in Sec. VI. Finally, concluding remarks are given in Sec. VII.

\section{MATHEMATICAL MODEL}

The dynamics of quantum ion acoustic waves in electron-ion plasmas is here studied using the following hydrodynamic model [54] which includes the ion continuity equation,

$$
\frac{\partial n_{i}}{\partial t}+\nabla \cdot\left(n_{i} \mathbf{u}_{i}\right)=0
$$

the momentum equation for the cold ions,

$$
m_{i}\left[\frac{\partial \mathbf{u}_{i}}{\partial t}+\left(\mathbf{u}_{i} \cdot \nabla\right) \mathbf{u}_{i}\right]=-e \nabla \phi
$$

the momentum equation for the inertialess electrons,

$$
0=e \nabla \phi-\frac{\nabla P_{e}}{n_{e}}+\frac{\xi \hbar^{2}}{6 m_{e}} \nabla\left(\frac{\Delta \sqrt{n_{e}}}{\sqrt{n_{e}}}\right)
$$

and Poisson's equation,

$$
\Delta \phi=4 \pi e\left(n_{e}-n_{i}\right)
$$

in which $n_{e}, n_{i}, \mathbf{u}_{i}, P_{e}$ and $\phi$ are the electron number density, ion number density, ion fluid velocity, electron fluid pressure, and electrostatic potential. Other parameters have their usual meanings. The parameter $\xi$ is a correction for low-frequency $\left(\omega \ll k V_{F}\right.$ with $V_{F}$ being the electron Fermi speed) wave phenomena to the Bohm force in the hydrodynamic formulation, defined as

$$
\xi=\frac{\operatorname{Li}_{3 / 2}\left[-\exp \left(\beta \mu_{0}\right)\right] \mathrm{Li}_{-1 / 2}\left[-\exp \left(\beta \mu_{0}\right)\right]}{\operatorname{Li}_{1 / 2}\left[-\exp \left(\beta \mu_{0}\right)\right]^{2}},
$$


where $\beta=1 / k_{B} T$ with $T$ being the electron temperature, $\mu_{0}$ denotes the equilibrium chemical potential of the electron fluid, and $\operatorname{Li}_{\nu}(z)$ is the polylogarithm function of order $\nu$ and argument $z$. The polylogarithm function has the integral form

$$
\operatorname{Li}_{\nu}\left(-\mathrm{e}^{z}\right)=-\frac{1}{\Gamma(\nu)} \int_{0}^{\infty} \frac{x^{\nu-1}}{\exp (x-z)+1} \mathrm{~d} x, \quad \nu>0,
$$

where $\Gamma$ is the gamma function. For a given value of $T$, the value of $\xi$ decreases from the limiting classical value of $\xi=1$ for $\beta \mu_{0} \ll-1$ to the limiting fully degenerate value $\xi=1 / 3$ for $\beta \mu_{0} \gg 1$ in which limit $\mu_{0} \simeq E_{F}$ where $E_{F}=k_{B} T_{F}$ is the Fermi energy of the system and $T_{F}$ is the Fermi temperature, dependent only on the electron number density. Note that in the fully degenerate limit, $z \gg 1$, we have $\lim _{z \rightarrow \infty} \operatorname{Li}_{\nu}\left(-e^{z}\right)=-z^{\nu} / \Gamma(\nu+1)$ and in the classical limit, $z \ll-1$, we have $\operatorname{Li}_{\nu}\left(-e^{z}\right) \approx-e^{z}$.

A new generalized quantum hydrodynamic formalism based on density functional theory (DFT) has recently appeared, which takes into account both the Hartree mean-field and electron exchange-correlation potentials in an integral form [55, 56] as a function of the local electron number density. Moreover, a density functional exchange-correlation potential [57] may be easily incorporated in the current hydrodynamic model (1) as has been done in recent literature [58-60]. However, investigations based on kinetic theory [61] reveals that such a time-independent density functional exchange contribution to hydrodynamic formalism may give misleading results for low phase speed phenomena such as ion acoustic oscillations and may only provide reasonable results for fast electron plasma excitations. It is also noted that the exchange-correlation contribution to many-body system is independent of the single-particle orbital but depends only on the local fluid density [55].

For an isothermal electron fluid, the equation of state may be written in the form [54]

$$
n_{e}=-N \operatorname{Li}_{3 / 2}[-\exp (\beta \mu)], \quad P_{e}^{(i s)}=-\frac{N}{\beta} \operatorname{Li}_{5 / 2}[-\exp (\beta \mu)],
$$

where the effective density of states of the electrons is given by [36]

$$
N=\frac{2}{\Lambda_{e}^{3}}=2\left(\frac{m_{e}}{2 \pi \beta \hbar^{2}}\right)^{3 / 2},
$$

where $\Lambda_{e}$ is the electron thermal de Broglie wavelength. The isothermal equation of state (4) may be written in the following compact form

$$
P_{e}^{(i s)}=\frac{n_{e}}{\beta} \frac{\operatorname{Li}_{5 / 2}[-\exp (\beta \mu)]}{\operatorname{Li}_{3 / 2}[-\exp (\beta \mu)]} .
$$


Note that in the classical limit one arrives at $P_{e}^{(i s)}=n_{e} k_{B} T$ and in the complete degeneracy limit $P_{e}^{(i s)}=(2 / 5) n_{e} k_{B} T_{F}$. The degeneracy parameter $\delta=T_{F} / T$ is a measure of the degree of degeneracy of the electron fluid with the limits $\delta \ll 1$ and $\delta \gg 1$ corresponding to the classical and fully degenerate cases, respectively. Therefore, the model is valid for electronion plasma with a wide range of non-relativistic degrees of degeneracy.

On the other hand, the one-dimensional adiabatic equation of state of electrons may be written as [68]

$$
P_{e}^{(a d)}=\frac{n_{e 0} \mathrm{Li}_{5 / 2}\left[-\exp \left(\beta \mu_{0}\right)\right]}{\beta \mathrm{Li}_{3 / 2}\left[-\exp \left(\beta \mu_{0}\right)\right]}\left(\frac{n_{e}}{n_{e 0}}\right)^{3},
$$

In the classical limit for adiabatic equation of state one obtains $P_{e}^{(a d)}=\left(n_{e} / n_{e 0}\right)^{3} n_{e} k_{B} T$ which corresponds to the classical electron gas with the adiabatic index $\gamma=3$ in a gas of degree of freedom $D=1$ with $\gamma=(D+2) / D$. In the fully degenerate limit one has $P_{e}^{(a d)}=(2 / 5)\left(n_{e} / n_{e 0}\right)^{3} n_{e} k_{B} T_{F}$ where $n_{e 0}$ is the equilibrium electron number density.

\section{ENERGY SPECTRUM OF QUASIPARTICLE IN A BOX}

Using the Madelung transformations $\mathcal{N}=\Psi(\mathbf{r}, t) \exp [i S(\mathbf{r}, t) / \hbar]$ with $\Psi(\mathbf{r}, t)=\sqrt{n(\mathbf{r}, t)}$ and $\nabla S(\mathbf{r}, t)=m_{e} \mathbf{u}_{i}$ the hydrodynamic set of equations (1) may in the quasineutrality limit $\left(n_{e} \simeq n_{i}=n\right)$ be cast into the effective Schrödinger equation [43]

$$
i \hbar \frac{\partial \mathcal{N}}{\partial t}=-\frac{\eta \hbar^{2}}{2 m_{e}} \Delta \mathcal{N}+\alpha \mu \mathcal{N}
$$

where $\alpha=m_{e} / m_{i}$ and $\eta=\alpha \xi / 3$. Note that in obtaining Eq. (8) for low-frequency free ion-acoustic oscillations, we applied the isothermal equation of state for electrons (6) via the simplifying identity $\nabla P=n \nabla \mu$ which can be readily confirmed using definitions (4). In normalized units $\left(\Psi \rightarrow \Psi / \sqrt{n_{0}}\right.$ ) we can write a one-dimensional version of Eq. (8) as

$$
i \frac{\partial \mathcal{N}}{\partial t}=-\eta \frac{\partial^{2} \mathcal{N}}{\partial x^{2}}+\alpha \mu \mathcal{N}
$$

in which $\mu$ is normalized to the plasmon energy $E_{p}=\hbar \omega_{p}$ with $\omega_{p}=\sqrt{4 \pi e^{2} n_{0} / m_{e}}$ being the electron plasma frequency, and the time and space coordinates are respectively normalized by $1 / \omega_{p}$ and $\lambda_{p}$, in which $\lambda_{p}=2 \pi / k_{p}$ is the plasmon length with $k_{p}=\sqrt{2 m_{e} E_{p}} / \hbar$ being the plasmon wavenumber. We first consider linear ion quasi-particle excitations in which the electron fluid constitutes a homogeneous isothermal gas with the equilibrium chemical 
potential $\mu \simeq \mu_{0}$ in which the singly ionized ions oscillate with frequency $\omega \ll k V_{F}$ with $V_{F}$ being the Fermi speed. On the other hand, for standing waves with $S=S(t)\left(u_{x}=0\right)$, Eq. (9) may be decomposed into the following linear system using the separation of variables $\mathcal{N}(x, t)=\Psi(x) \exp (-i \omega t)$

$$
\frac{d^{2} \Psi}{d x^{2}}+k^{2} \Psi=0, \quad k=\sqrt{\frac{\omega-\alpha \mu_{0}}{\eta}}
$$

where $\omega$ is the normalized quasi-particle (a quasi-particle of ion excitations in a periodic crystal lattice is called a phonon) eigenfrequency. It gives the plane wave solution for the wave function of a free quasi-particle

$$
\mathcal{N}(x, t)=A \exp (i k x-i \omega t)
$$

Note that, unlike plasmon excitations which are two-tone oscillations, there is only one scalelength associated with the ion quasi-particle. Equation (9) may be generalized to include an external potential $\Phi(x)$ as compared to the internal potential $\mu$

$$
i \frac{\partial \mathcal{N}}{\partial t}=-\eta \frac{\partial^{2} \mathcal{N}}{\partial x^{2}}+[\alpha \mu+\Phi(x)] \mathcal{N}
$$

Let us now consider the energy levels of linear excitations in a hard wall confining potential well of width $l$ (where $l$ is the plasma dimension), i.e. $\Phi(0<x<l)=0$ and $\Phi(x \geq l, x \leq$ $0)=\infty$. Analogous to standard quantum mechanics problem of particle in a box one obtains the normalized wavefunction and quantized wavenumbers

$$
\mathcal{N}(x)=\sin (k x) \exp (-i \omega t), \quad k=\frac{\pi q}{l}
$$

where $q$ is an integer. Note that the spatial variation in the electron (and ion) number density is obtained from $n=\mathcal{N N}^{*}=\sin ^{2}(\pi q x / l)$. The quantized eigenfrequencies of the quasi-particles are

$$
\omega=\alpha \mu_{0}+\frac{\eta \pi^{2} q^{2}}{l^{2}}
$$

It is noted that the eigenfrequency $\omega$ is limited from below to $\alpha \mu_{0}+\eta \pi^{2} / l^{2}$ which clearly depends on the equilibrium chemical potential of the ambient electron gas. Note also that (13) reduces to the plasmon energy eigenfrequencies [10] by taking $\alpha=1$ and $\xi=3$ appropriate for plasmon oscillations [54]. In the high frequency linear limit, by using the quasi-particle frequency spectrum, one can calculate some useful macroscopic physical properties such 
as the heat capacity, optical response, and thermal expansion for plasmas with arbitrary degrees of electron degeneracy.

Collective plasma-ion excitations (quasi-particles) are quantum mechanical analogue of phonons which are due to lattice vibrations in solids. Phonons are known to play important role in heat capacity and heat transport in solids. For instance, there can be two sources of heat capacity in plasmas. The important one is due to electron plasma oscillations which has recently been treated in Ref. [63]. The other contribution comes from the quasi-particle excitations. Hence, from the quantized energy spectrum (14) of quasi-particles in quantum plasmas one can calculate the quasi-particle density of states (DoS) quite similar to those for fermions in metals. The quasi-particle energy spectrum and DoS is however closely related to the energy level of electrons in metals due to the fact that collective ion dynamics in a plasma is coupled with the electron fluid motion via the electrostatic perturbations (e.g. see Eq. (1)). Now taking the normalized parabolic energy dispersion $E=\epsilon-\alpha \mu_{0}=\eta k^{2} / 2$ for the Bosonic quasi-particles, the enumeration of available modes and taking into account the Pauli exclusion principle for electrons leads to the quasi-particle $\operatorname{DoS} D(E)=3 N /(2 \eta E)$ where $N$ is the number of electrons (ions). A rough estimate of the quasi-particle contribution to the specific heat in the low-temperature, quasineutral limit based on current model is

$$
C_{q p}=\int_{0}^{\infty} \frac{d f(E, T)}{d T} E D(E) d E
$$

with the bosonic occupation function $f(E, T)=1 /\left[\exp \left(E / k_{B} T\right)-1\right]$.

On the other hand, an analytic solution for the generalized equation (12) can be found using the WKB approximation [64], in the linear limit $\mu \simeq \mu_{0}$. In the classically allowed region, away from the turning points, the WKB solution reads

$$
\Psi(x)=\frac{C_{+} \exp \left[\frac{i}{\sqrt{\eta}} \int d x \sqrt{\omega-\alpha \mu_{0}-\Phi(x)}\right]+C_{-} \exp \left[\frac{-i}{\sqrt{\eta}} \int d x \sqrt{\omega-\alpha \mu_{0}-\Phi(x)}\right]}{\sqrt[4]{\left[\Phi(x)+\alpha \mu_{0}-\omega\right] / \eta}} .
$$

For instance, for plasma confined in a parabolic external potential $\Phi(x)=\omega_{0}^{2} x^{2}$, the approximate solution within the potential is

$$
\Psi(x)=\frac{C_{+} \exp [i \delta(x)]+C_{-} \exp [-i \delta(x)]}{\sqrt[4]{\left[\omega_{0}^{2} x^{2}+\alpha \mu_{0}-\omega\right] / \eta}},
$$

where

$$
\delta(x)=\frac{1}{2 \sqrt{\eta}}\left\{x \sqrt{\omega-\alpha \mu_{0}-\omega_{0}^{2} x^{2}}+\frac{\left(\omega-\alpha \mu_{0}\right)}{\omega_{0}} \tan ^{-1}\left[\omega_{0} x / \sqrt{\omega-\alpha \mu_{0}-\omega_{0}^{2} x^{2}}\right]\right\} .
$$




\section{WEAKLY NONLINEAR DRIVEN EXCITATIONS}

Let us now consider the quasi-particle excitations in the weakly nonlinear limit. Parametrically driven NLSE corresponding to (9) has the form [65-67]

$$
i \frac{\partial \mathcal{N}}{\partial t}+\eta \frac{\partial^{2} \mathcal{N}}{\partial x^{2}}-\alpha \mu(\Psi) \mathcal{N}=\Omega \exp \left(-i \omega_{d} t\right) \mathcal{N}^{*}
$$

where $\Omega$ and $\omega_{d}$ is the driving amplitude and frequency, respectively. Assuming a solution on the form $\mathcal{N}(x, t)=\Psi(x) \exp \left(-i \omega_{d} t / 2\right)$, the autonomous equation governing the motion of a quasi-particle can be written as

$$
\frac{d^{2} \Psi}{d x^{2}}+\left[\frac{\omega_{d} / 2-\alpha \mu(\Psi)-\Omega}{\eta}\right] \Psi=0
$$

The eigenfrequency and wave function of the quasi-particle in an infinite potential well of width $l$ (corresponding to the quasi-particle oscillating in the pseudo-potential), employing the driven $\operatorname{NLSE}(20)$ in the linear limit $\left(\mu \simeq \mu_{0}\right)$, is found to be $\omega_{d} / 2$ and $\mathcal{N}(x, t)=$ $A \exp \left(i K x-i \omega_{d} t / 2\right)$ with $K=\sqrt{\left(\omega_{d} / 2-\alpha \mu_{0}-\Omega\right) / \eta}$. It is interesting to note that stable driven quasi-particle excitations require that $\Omega<\omega_{d} / 2-\alpha \mu_{0}$, so that there is a distinct limit on maximum amplitude of stable driven quasi-particles. That is, for a given driver frequency its amplitude must exceed a critical value in order to parametrically excite a quasi-particle.

Two notable differences appear in the characteristics of driven quasi-particle excitations compared to the free ones, considered in Sec. V. The first is that the eigenfrequency of driven oscillations are shifted to higher values compared to those of free excitations. The second one is that the driven excited quasi-particle eigenfrequencies are exactly half of that corresponding to the driver frequency. In other words, the eigenfrequency spacings for parametrically driven quasi-particles in a potential width of length $l$ are twice of those for free quasi-particle in a hard box of the same length, i.e.,

$$
\omega_{d}=2 \Omega+2 \alpha \mu_{0}+\frac{2 \eta \pi^{2} q^{2}}{l^{2}}
$$

Moreover, it is possible to obtain approximate analytic solution to the linearized driven NLSE (19) in the presence of an arbitrary external potential, $\Phi(x)$ using the WKB approximation. Such a solution is given by (16) with the replacement $\omega \rightarrow \omega_{d} / 2-\Omega$.

Here a discussion on the validity of the above considered linearized NLSE and driven NLSE models is in order. While the bold assumption $\mu \simeq \mu_{0}$ may seem very liming, 
it is quite reasonable in fully degenerate plasmas such as liquid metals and nanometallic fluids to assume that the chemical potential stays essentially unchanged during collective ion excitations. In such systems in which the electron temperature is much less that the characteristic Fermi temperature. The electron temperature is not a characteristic parameter of the degenerate system anymore and the chemical potential $\mu$ is replaced with a constant Fermi energy which is solely a function of the electron number density. Therefore, the dynamics of ion oscillations in fully degenerate plasmas which are ubiquitous in nature are ruled by the nearly fixed Fermi energy. Moreover, as the number density of the electrons decreases and the plasma becomes partially degenerate, such as in semiconductors, the temperature becomes a fundamental parameter of ion and electron dynamic processes and the changes in chemical potential cannot be ignored.

It is easily confirmed that Eq. (20) admits the following first integral

$$
\frac{1}{2}\left(\frac{d \Psi}{d x}\right)^{2}+V(\Psi)=E^{\prime}
$$

in which $E^{\prime}$ is the energy eigenvalue of the quasi-particle [62] and the pseudo-potential corresponding to this Hamiltonian reads

$$
U(\Psi)=E^{\prime}-V(\Psi)=E^{\prime}-\frac{\alpha}{\eta} \int_{1}^{\Psi} \mu(\Psi) \Psi d \Psi-\left(\frac{\omega_{d} / 2-\Omega}{2 \eta}\right)\left(\Psi^{2}-1\right) .
$$

Using the expansion of the chemical potential around the equilibrium value, we find

$$
V(\Psi)=-\frac{\alpha}{\eta} \int_{1}^{\Psi}\left[\mu_{0}+\left.(\Psi-1) \frac{\partial \mu}{\partial \Psi}\right|_{\Psi=1}\right] \Psi d \Psi-\left(\frac{\omega_{d} / 2-\Omega}{2 \eta}\right)\left(\Psi^{2}-1\right),
$$

which is put in a simplified form of a Helmholtz potential as

$$
V(\Psi)=-\frac{c}{2} \Psi^{2}+\frac{d}{3} \Psi^{3}+V_{0}
$$

where $V_{0}$ is an arbitrary reference potential denoting the equilibrium point and the parameters $c$ and $d$ are respectively the dispersion and nonlinear coefficients given by

$$
c=d+\left(\frac{\omega_{d} / 2-\Omega+2 \alpha \mu_{0}}{2 \eta}\right), \quad d=-\left.\frac{2 \alpha}{\eta} \frac{\partial \mu}{\partial n}\right|_{n=1}=-\left(\frac{2 \alpha \theta}{\eta}\right) \frac{\operatorname{Li}_{3 / 2}\left[-\exp \left(\mu_{0} / \theta\right)\right]}{\operatorname{Li}_{1 / 2}\left[-\exp \left(\mu_{0} / \theta\right)\right]} .
$$

where $\theta=T / T_{p}$ with $T_{p}=E_{p} / k_{B}$ being the characteristic plasmon temperature. Taking $V_{0}=c / 2-d / 3$ brings the equilibrium point $\Psi=1$ to the origin which is more appropriate for 
analytic purpose. The pseudo-potential (25) has another extremum value at $\Psi_{m}=c / d$ given by $V_{m}=-c^{3} /\left(6 d^{2}\right)$. The energy $E^{\prime}$ of quasi-particle oscillating extreme between points $\Psi_{2}$ and $\Psi_{3}$ which are roots of $U(\Psi)=0$ can vary in the range $0<E^{\prime}<V_{m}$. The quasi-particles range from very high frequency, low amplitude linear excitations with energy $E^{\prime} \rightarrow 0$ up to the low frequency, high amplitude cnoidal and soliton quasi-particles which appear as Faraday ripples for $E^{\prime} \rightarrow V_{m}$.

The solution to the energy equation is given by the integral form

$$
x-x_{0}=\int_{\Psi_{2}}^{\Psi_{3}} \frac{d \Psi}{\sqrt{2 U(\Psi)}}
$$

where $x_{0}$ is the reference point of the oscillations and $\Psi_{2}$ and $\Psi_{3}>\Psi_{2}$ are the extreme points between which the quasi-particle oscillates in the corresponding pseudo-potential. The integral (28) can be exactly solved in the current weakly nonlinear limit (24). Taking $x_{0}=V_{0}=0$, leads to the exact analytic solution [62]

$$
\Psi(x)=\Psi_{2}+\left(\Psi_{3}-\Psi_{2}\right) \mathrm{cn}^{2}\left[k^{\prime}\left(x-x_{0}\right), m\right], \quad k^{\prime}=\sqrt{\frac{d\left(\Psi_{3}-\Psi_{1}\right)}{6}}, \quad m=\frac{\Psi_{3}-\Psi_{2}}{\Psi_{3}-\Psi_{1}},
$$

where cn is the Jacobi-elliptic function with $\Psi_{3} \geq \Psi_{2} \geq \Psi_{1}$ being the crossing points of the pseudo-potential $V(\Psi)$ at pseudo-energy level $E^{\prime}$ and $0 \leq m \leq 1$ is the modulus of Jacobicn function having the limiting values $m=0$ and $m=1$ which correspond respectively to sinusoidal and solitary excitations. The roots $\Psi_{i}$ are given in terms of the potential parameters $(26)$ as

$$
\begin{aligned}
& \Psi_{1}=\frac{1}{8}\left(\frac{c^{2}}{d}-\frac{c^{4}}{d Z}+\frac{Z}{d}\right) \\
& \Psi_{2,3}=\frac{c^{2}}{8 d}+\frac{(1 \mp i \sqrt{3}) c^{4}}{16 d Z}+\frac{(1 \mp i \sqrt{3}) Z}{16 d} \\
& Z=\left(16 \sqrt{6} \sqrt{c^{6} d^{2} E^{\prime}+384 d^{4} E^{\prime 2}}-768 d^{2} E^{\prime}-c^{6}\right)^{1 / 3} .
\end{aligned}
$$

The Jacobi-cn elliptic function has the limiting forms $\mathrm{cn}(\mathrm{x}, 0)=\cos (\mathrm{x})$ and $\mathrm{cn}(\mathrm{x}, 1)=\operatorname{sech}(\mathrm{x})$. Also, In the sinusoidal excitation limit we have $\Psi_{2} \simeq \Psi_{3}$, while in the soliton limit $\Psi_{1} \simeq \Psi_{2}$ [62]. The weakly nonlinear driven excitations studied in this section may also be extended to the case with the hard wall rectangular potential well of length $l$ similar to the case of linear excitations. In that case the quantization of weakly nonlinear excitation in the pseudo-potential consequently leads to quantization of the eigenfrequencies and amplitude 
of driver via descretization of the pseudo-energy $E^{\prime}$. Since, the cn-function $\mathrm{cn}^{2}$ has a period $\tau=2 K(m)$ with $K(m)$ being the complete elliptic integral of first kind, the quantization condition for the weakly nonlinear quasi-particle excitations readily become $k^{\prime}=2 q^{\prime} K(\mathrm{~m}) / l$ with $q^{\prime}$ being an integer characterizing the quantum number. It is clear that the quantization condition is only satisfied for specific values of the driver frequency $\omega_{d}$ and its amplitude $\Omega$ which are parametrically related to the energy $E^{\prime}$ of the quasi-particle.

\section{FULLY NONLINEAR DRIVEN EXCITATIONS}

In order to study fully nonlinear driven Faraday quasi-particle excitations let us again use the isothermal equation of state for the electron fluid, since it is assumed that the driving frequency is much lower than the Fermi frequency of the electrons, $\omega_{F}=k_{F} V_{F}$, in which $k_{F}$ and $V_{F}$ are Fermi wave vector and speed, respectively. The fully nonlinear driven NLSE governing these excitations is given by Eq. (19). This equation admits the first integral

$$
\frac{1}{2}\left(\frac{d \Psi}{d x}\right)^{2}+V(\Psi)=0, \quad U(\Psi)=E^{\prime \prime}-V(\Psi)
$$

in which $E^{\prime \prime}$ is the energy eigenvalue of the corresponding quasi-particle. Appropriate integration of the pseudo-force (30) leads to the pseudo-potential

$$
U(\Psi)=E^{\prime \prime}-V(\Psi)=E^{\prime \prime}-\frac{\alpha}{\eta} \int_{1}^{\Psi} \mu(\Psi) \Psi d \Psi-\left(\frac{\omega_{d} / 2-\Omega}{2 \eta}\right)\left(\Psi^{2}-1\right)-V_{0},
$$

Therefore the analytical full form of pseudo-potential is given by

$$
V(\Psi)=\frac{\alpha}{2 \eta}\left\{\left(\mu-\mu_{0}\right) \Psi^{2}-\left[P_{e}^{(i s)}(\mu)-P_{e}^{(i s)}\left(\mu_{0}\right)\right]\right\}+\left(\frac{\omega_{d} / 2-\Omega}{2 \eta}\right)\left(\Psi^{2}-1\right)+V_{0},
$$

where we have

$$
P_{e}^{(i s)}(\mu)=\frac{\theta \operatorname{Li}_{5 / 2}[-\exp (\mu / \theta)]}{\operatorname{Li}_{3 / 2}[-\exp (\mu / \theta)]} \Psi^{2}, \quad P_{e}^{(i s)}\left(\mu_{0}\right)=\frac{\theta \operatorname{Li}_{5 / 2}\left[-\exp \left(\mu_{0} / \theta\right)\right]}{\operatorname{Li}_{3 / 2}\left[-\exp \left(\mu_{0} / \theta\right)\right]},
$$

The pseudo-potential (32) is complicated to handle analytically, since, the chemical potential $\mu$ is a function of $\Psi$. However, in the fully degenerate limit where $\mu / \theta \gg 1$ we have $P_{e}^{(i s)}(\Psi)=2 \delta_{0} \Psi^{2} / 5$ and $\mu=E_{F}=\delta_{0} \Psi^{4 / 3}$ where $\delta_{0}=T_{F 0} / T_{p}$ is the normalized equilibrium value of the Fermi temperature. The pseudo-potential for Faraday waves of ion excitations in a fully degenerate electron gas is

$$
V(\Psi)=\frac{\alpha \delta_{0}}{2 \eta}\left[\left(\Psi^{4 / 3}-1\right) \Psi^{2}-\frac{2}{5}\left(\Psi^{2}-1\right)\right]+\left(\frac{\omega_{d} / 2-\Omega}{2 \eta}\right)\left(\Psi^{2}-1\right)+V_{0} .
$$


The nonlinear solution, which can only be evaluated numerically, is given by the integral $[62]$

$$
x-x_{0}=\int \frac{d \Psi}{\sqrt{2 U(\Psi)}}
$$

The pseudo-potential (32) (taking $V_{0}=0$ ) has an equilibrium value $V_{e q}=0$ corresponding to the rest point of the quasi-particle at $\Psi=1$ and a maximum value $V_{m}$ corresponding to $\Psi_{m}$ which is the point at which the pseudo-potential maximizes. The pseudo-frequency eigenvalues of quasi-particle for given plasma parameters varies in the range $0<E^{\prime \prime}<$ $V_{m}$ with the lower/upper limit corresponding to the sinusoidal/soliton excitation pseudoenergy. Note that the roots $\Psi_{i}$ in this case follow the same ordering as before except that $\Psi_{1}<0$ in this case. The quantization condition in this case becomes $l=q^{\prime \prime} \Lambda$ in which $\Lambda$ is the wavelength of an arbitrary-amplitude quasi-particle and $q^{\prime \prime}$ is an arbitrary integer characterizing the quantum number. The wavelength $\Lambda$ is defined as

$$
\Lambda=2 \int_{\Psi_{2}}^{\Psi_{3}} \frac{d \Psi}{\sqrt{2 U(\Psi)}}
$$

in which $\Psi_{2}$ and $\Psi_{3}$ are the roots of $U(\Psi)=0$. Only for quantized values of $E^{\prime \prime}$ for given driving frequency $\omega$ and amplitude $\Omega$ for electron gas of chemical potential $\mu_{0}$ at temperature $T$, the condition $l=q^{\prime \prime} \Lambda$ is satisfied. It is, however, concluded that the linear excitation quantization of the Schrödinger equation when $E^{\prime \prime} \rightarrow 0$ is a very special case of the broader nonlinear Faraday quantization phenomena described by the driven NLSE in the full range $0 \leq E^{\prime \prime} \leq V_{m}$

It is to be noted that the violation of quasineutrality in the very high temperature limit may break down the validity of the current microscopic Faraday wave model. For metals and semiconductors which are strongly coupled plasmas with the quantum coupling parameter exceeding unity, the effective ionic correlations lead to crystalline structure formation with very high binding energies. In such as case the application of an RF source for high amplitude ion fluid Faraday excitations may lead to excessive heat production causing phase transitions and instabilities in the specimen. There are, however, a variety of liquid metals such as mercury, caesium, rubidium, francium, gallium, and gallium-based alloys with low pressure and temperature melting point or variety of metal-based electrolytes for which Faraday wave experiment may be applicable. For these cases, a simple experimental setup may be realized for atomic scale microscopic large-amplitude Faraday wave excitations. A flat RF 
transparent cylindrical container with variable height directed at $z$ axis is filled with a liquid metal such as caesium with melting point of 28.5 degree Celsius or rubidium with melting point 39 degree Celsius. The external low radio-frequency (LRF) excitation with energy band $E \simeq \sqrt{m_{e} / m_{i}} E_{p}$ in which $E_{p}=\hbar \omega_{p}$ is the electron plasma frequency is axially applied to the specimen. Note that the plasmon energy for caesium and rubidium are respectively $2.9 \mathrm{eV}$ and $3.4 \mathrm{eV}$. Then a horizontal direct current (DC) probe may be used to study the Faraday quantization effect. Moreover, the existence of fluid viscosity is essential and a key parameter in production of Faraday patters on a liquid surface. In quantum plasmas however the viscosity comes from weak ion correlations in liquid metals which may change in an ionic metal-based electrolyte by metal concentrations. Then the viscous damping of the fluid, which is considered in Sec. VI, can be experimentally tuned to the desired value.

\section{PARAMETRICALLY DRIVEN DAMPED EXCITATIONS}

Finally, let us consider parametrically driven damped excitations of ions in arbitrary degenerate plasmas. The normalized NLSE including the parametric driving force as well as the damping term may be written as [65]

$$
i \frac{\partial \mathcal{N}}{\partial t}+\eta \frac{\partial^{2} \mathcal{N}}{\partial x^{2}}-\alpha \mu \mathcal{N}+i \gamma \mathcal{N}=\Omega \exp \left(-i \omega_{d} t\right) \mathcal{N}^{*}
$$

in which the last term in the left-hand side accounts for the dissipative losses. Physically, the dissipative damping effect arises due to electron-ion collisions in the plasma. Assuming a wave function of form $\mathcal{N}(x, t)=\Psi(x) \exp \left[\left(-i \omega_{d} / 2-\gamma\right) t\right],(\gamma>0)$ we arrive at the following autonomous equation

$$
\frac{d^{2} \Psi}{d x^{2}}+\left(\frac{\omega_{d} / 2-\alpha \mu-\Omega}{\eta}\right) \Psi=0
$$

Note that Eq. (38) is identical to Eq. (20). In the linear limit $\mu \simeq \mu_{0}$ we find a temporally decaying plane-wave solution with the damping rate $\gamma$

$$
\mathcal{N}(x, t)=A \exp \left(i k x-i \omega_{d} t / 2\right) \exp (-\gamma t), \quad k=\sqrt{\frac{\omega_{d} / 2-\alpha \mu_{0}-\Omega}{\eta}} .
$$

It is seen that (39) is a transient solution since at $t \rightarrow \infty$ the wave function and hence the number density $n=\mathcal{N N}^{*}$ vanishes. Therefore, the stable solution in the presence of driving force will be the sinusoidal one with half the frequency of the periodic driving force. 


\section{CONCLUSION}

In this paper we have studied the quantized frequency spectrum of linear and fully nonlinear quasi-particle excitations in a quasineutral electron-ion plasma within the framework of quantum hydrodynamic model. The set of hydrodynamic equations is reduced to an effective nonlinear Schrödinger equation (NLSE) from which the quantized frequency spectrum of quasi-particle excitations is obtained. It was shown that the quantized frequency levels of linear quasi-particle excitations confined in a rectangular potential well of length $l$ is similar to that of a single electron confined in same infinite potential well except for much reduced level spacings for ion quasi-particles which is proportional to the electron to ion mass-ratio. Several basic physical properties of quasineutral quantum plasmas such as the ion quasi-particle contribution to heat capacity and thermal expansion can be directly calculated using the obtained energy spectrum at low temperature limit. We also investigated the quantized frequency spectrum of weakly and fully nonlinear arbitrary amplitude quantum Faraday excitations using the pseudo-potential method.

[1] M. Faraday, On a peculiar class of acoustical figures; and on certain forms assumed by groups of particles upon vibrating elastic surfaces, Philos. Trans. R. Soc. London 121, 299 (1831).

[2] M. Torres, G. Pastor, I. Jimnez, and F.Montero De Espinosa, Five-fold quasicrystal-like germinal pattern in the Faraday wave experiment, Chaos, Solitons Fractals 5, 2089 (1995).

[3] W. S. Edwards and S. Fauve, Patterns and quasi-patterns in the Faraday experiment, J. Fluid Mech. 278, 123 (1994).

[4] B. A. Puthenveettil and E. J. Hopfinger, Evolution and breaking of parametrically forced capillary waves in a circular cylinder, J. Fluid Mech. 633, 355 (2009).

[5] E. Bodenschatz, W. Pesch, and G. Ahlers, Recent developments in Rayleigh-Bnard convection, Annu. Rev. Fluid Mech. 32, 709 (2000).

[6] G. I. Taylor, Stability of a viscous liquid contained between two rotating cylinders, Philos. Trans. R. Soc. London 223, 289 (1923).

[7] X. Zhao, J. Tang, and J. Liu, Phys. Rev. Fluids 3, 124804(2018).

[8] S. Alazemi, W. Lacarbonara, and M. F. Daqaq, J. Appl. Phys. 122, 224501 (2017); 
doi.org/10.1063/1.4999834

[9] M. Bonitz, D. Semkat, A. Filinov, V. Golubnychyi, D. Kremp, D. O. Gericke, M. S. Murillo, V. Filinov, V. Fortov, W. Hoyer, Theory and simulation of strong correlations in quantum Coulomb systems, J. Phys. A 365921 (2003). doi:10.1088/0305-4470/36/22/313

[10] M. Akbari-Moghanjoughi, Quantized plasmon excitations of electron gas in potential well, Phys. Plasmas 26, 012104 (2019). doi:10.1063/1.5078740

[11] S. Chandrasekhar, An Introduction to the Study of Stellar Structure, (The University of Chicago Press, Chicago, 1939).

[12] F. Hoyle and W. A. Fowler, Nucleosynthesis in Supernovae, Astrophys. J. 132, 565 (1960). doi:10.1086/146963

[13] E. Madelung, Quantentheorie in hydrodynamischer Form, Z. Phys. 40, 322 (1926). doi:10.1007/BF01400372

[14] D. Bohm and D. Pines, A Collective Description of Electron Interactions: III. Coulomb Interactions in a Degenerate Electron Gas, Phys. Rev. 92609 (1953). doi:10.1103/PhysRev.92.609

[15] D. Pines and D. Bohm, A Collective Description of Electron Interactions: II. Collective vs. Individual Particle Aspects of the Interactions, Phys. Rev. 85, 338 (1952).

[16] P. Levine and O. V. Roos, Plasma Theory of the Many-Electron Atom, Phys. Rev. 125, 207 (1962). doi:10.1103/PhysRev.125.207

[17] Y. Klimontovich and V. P. Silin, The Spectra of Systems of Interacting Particles, in Plasma Physics, edited by J. E. Drummond (McGraw-Hill, New York, 1961; Dover Publications Inc. Mineola, New York, 2013).

[18] M. Marklund and G. Brodin, Dynamics of Spin-1/2 Quantum Plasmas. Phys. Rev. Lett. 98, 025001 (2007). doi:10.1103/PhysRevLett.98.025001

[19] N. Crouseilles, P. A. Hervieux, and G. Manfredi, Quantum hydrodynamic model for the nonlinear electron dynamics in thin metal films, Phys. Rev. B 78, 155412 (2008). doi:10.1103/PhysRevB.78.155412

[20] G. Brodin, M. Marklund, and G. Manfredi, Quantum Plasma Effects in the Classical Regime, Phys. Rev. Lett. 100, 175001 (2008). doi:10.1103/PhysRevLett.100.175001

[21] M. Marklund, G. Brodin, L. Stenflo, and C. S. Liu, New quantum limits in plasmonic devices, Europhys. Lett. 84, 17006 (2008). doi:10.1209/0295-5075/84/17006

[22] Y.-D. Jung, Semiclassical transition probabilities for the electron-impact excitation of hydro- 
genic ions in dense plasmas, Phys. Plasmas 2, 332 (1995). doi:10.1063/1.871440

[23] Y.-D. Jung, Quantum-mechanical effects on electron-electron scattering in dense hightemperature plasmas, Phys. Plasmas 8, 3842 (2001). doi:10.1063/1.1386430

[24] A. E. Dubinov and I. N. Kitayev, Two-tone nonlinear electrostatic waves in the quantum electron-hole plasma of semiconductors, Semiconductors 51, 14 (2017). doi:10.1134/S1063782617010079

[25] P. K. Shukla and B. Eliasson, Colloquium: Nonlinear collective interactions in quantum plasmas with degenerate electron fluids, Rev. Mod. Phys. 83, 885 (2011). doi:10.1103/RevModPhys.83.885

[26] P. K. Shukla and L. Stenflo, Dispersion relations for electromagnetic waves in a dense magnetized plasma, J. Plasma Phys. 74, 719 (2008). doi:10.1017/S0022377808007344

[27] P. K. Shukla and B. Eliasson, Novel Attractive Force between Ions in Quantum Plasmas, Phys. Rev. Lett. 108, 219902 (E) (2012). doi.org/10.1103/PhysRevLett.108.165007; Errata: Phys. Rev. Lett. 108, 219902 (2012); Phys. Rev. Lett. 109, 019901 (2012).

[28] B. Eliasson and M. Akbari-Moghanjoughi, Finite temperature static charge screening in quantum plasmas, Phys. Lett. A 380, 2518 (2016). doi:10.1016/j.physleta.2016.05.043.

[29] M. Marklund and P. K. Shukla, Nonlinear collective effects in photon-photon and photonplasma interactions, Rev. Mod. Phys. 78, 591 (2006). doi:10.1103/RevModPhys.78.591

[30] G. Brodin and L. Stenflo, Nonlinear dynamics of a cold collisional electron plasma, Phys. Plasmas 24, 124505 (2017). doi:10.1063/1.5011299

[31] A. Bret, Filamentation instability in a quantum plasma, Phys. Plasmas 14, 084503 (2007). doi:10.1063/1.2759886

[32] P. K. Shukla and B. Eliasson, Screening and wake potentials of a test charge in quantum plasmas, Phys. Lett. A, 372, 2897 (2008). doi:10.1016/j.physleta.2007.12.067

[33] M. Akbari-Moghanjoughi, Generalized sheath criterion for arbitrary degenerate plasmas, Phys. Plasmas 24, 012113 (2017). doi:10.1063/1.4975078

[34] J. Hurst, K. Lévêque-Simon, P. A. Hervieux, G. Manfredi and F. Haas, High-harmonic generation in a quantum electron gas trapped in a nonparabolic and anisotropic well, Phys. Rev. B 93, 205402 (2016). doi.org/10.1103/PhysRevB.93.205402

[35] H. Haug and S. W. Koch, Quantum Theory of the Optical and Electronic Properties of Semiconductors (World Scientific, Singapore, 2004). 
[36] C. Hu, Modern Semiconductor Devices for Integrated Circuits (Prentice Hall, Upper Saddle River, New Jersey, 2010) 1st ed.

[37] K. Seeger, Semiconductor Physics (Springer, Berlin, 2004) 9th ed.

[38] P. A. Markovich, C.A. Ringhofer, and C. Schmeister, Semiconductor Equations (Springer, Berlin, 1990).

[39] G. Manfredi, Preface to Special Topic: Plasmonics and solid state plasmas, Phys. Plasmas 25, 031701 (2018). doi:10.1063/1.5026653

[40] G. Manfredi, How to model quantum plasmas, Fields Inst. Commun. 46, 263287 (2005); in Proceedings of the Workshop on Kinetic Theory (The Fields Institute, Toronto, Canada 2004). arXiv:quant-ph/0505004.

[41] F. Haas, Quantum Plasmas: An Hydrodynamic Approach (Springer, New York, 2011).

[42] P. K. Shukla, B. Eliasson, Nonlinear aspects of quantum plasma physics, Phys. Usp. 51, 53 (2010). doi:10.3367/UFNe.0180.201001b.0055

[43] G. Manfredi and F. Haas, Self-consistent fluid model for a quantum electron gas, Phys. Rev. B 64, 075316 (2001). doi:10.1103/PhysRevB.64.075316

[44] M. Akbari-Moghanjoughi, A coupled pseudoforce model for quantum plasmon excitations, Phys. Plasmas 25, 102105 (2018). doi:10.1063/1.5055372

[45] C. Sulem and P.-L. Sulem, The Nonlinear Schrödinger Equation: Self-Focusing and Wave Collapse, Applied Mathematical Sciences, vol. 139 (Springer Verlag, New York, 1999).

[46] A. Hasegawa, Stimulated Modulational Instabilities of Plasma Waves, Phys. Rev. A 1, 1746 (1970). doi:10.1103/PhysRevA.1.1746

[47] V. E. Zakharov, Zh. Eksp. Teor. Fiz. 62, 1745 (1972).

[48] N. R. Pereira, Collisions between Langmuir solitons, Phys. Fluids, 20, 750 (1977). doi.org/10.1063/1.861947

[49] L. Stenflo, Nonlinear Equations for Acoustic Gravity Waves, Phys. Scr., 33, 156 (1986). doi:10.1088/0031-8949/33/2/010

[50] H. S. Chen and C. S. Liu, Solitons in nonuniform media, Phys. Rev. Lett 37, 693. doi:10.1103/PhysRevLett.37.693

[51] C. S. Liu, V. K. Tripathi and B. Eliasson, High-Power Laser-Plasma Interaction (Cambridge University Press, Cambridge, 2019). doi:10.1017/9781108635844

[52] G. J. Morales and Y. C. Lee, Ponderomotive-force effects in nonuniform plasma, Phys. Rev. 
Lett. 33, 1016 (1974). doi:10.1103/PhysRevLett.33.1016

[53] G. J. Morales and Y. C. Lee, Generation of density cavities and localized electric fields in a nonuniform plasma, Phys. Fluids 20, 1135 (1977). doi:10.1063/1.861675

[54] F. Haas and S. Mahmood, Linear and nonlinear ion-acoustic waves in nonrelativistic quantum plasmas with arbitrary degeneracy, Phys. Rev. E 92, 053112 (2015). doi:10.1103/PhysRevE.92.053112

[55] M. Bonitz, Zh. A. Moldabekov, and T. S. Ramazanov, Phys. Plasmas 26, 090601 (2019); doi.org/10.1063/1.5097885

[56] Zh. A. Moldabekov, M. Bonitz, and T. S. Ramazanov, Phys. Plasmas 25, 031903 (2018); doi.org/10.1063/1.5003910

[57] L. Hedin and B. I. Lundqvist, J. Phys. C: Solid State Phys. 4, 2064 (1971).

[58] K. Ourabah and M. Tribeche, Phys. Rev. E 88, 045101 (2013).

[59] S. Mahmood, H. Ur-Rehmana, S. Hussain, and M. Adnan, Phys. Lett. A, 383, 125840 (2019).

[60] K. Mebrouk, M. Tribeche, Phys. Lett. A, 378, 3523 (2014).

[61] G. Brodin, R. Ekman, and J. Zamanian, Phys. Plasmas, 26, 092113 (2019); doi: $10.1063 / 1.5104339$

[62] M. Akbari-Moghanjoughi, Energy spectrum of oscillations in generalized Sagdeev potential, Phys. Plasmas 24, 072107 (2017). doi:10.1063/1.4986224

[63] M. Akbari-Moghanjoughi, Heat capacity and electrical conductivity of plasmon excitations, Phys. Plasmas 26, 072106 (2019). doi:10.1063/1.5097144

[64] B. C. Hall, Quantum Theory for Mathematicians, Graduate Texts in Mathematics, vol. 267, (Springer, New York, 2013), ISBN 978-1461471158. doi:10.1007/978-1-4614-7116-5

[65] E. V. Zemlyanaya and I. V. Barashenkov, Traveling Solitons in the Damped-Driven Nonlinear Schrödinger Equation, Siam J. Appl. Math. 64, 800 (2004). doi:10.1137/S0036139903424837

[66] M. Akbari-Moghanjoughi, Traveling wave solutions of the nonlinear Schrödinger equation, Phys. Plasmas, 24, 102313 (2017). doi:10.1063/1.4999543

[67] M. Akbari-Moghanjoughi, Traveling wave solution of driven nonlinear Schrödinger equation, Phys. Plasmas, 24, 092117 (2017). doi:10.1063/1.4999783

[68] B. Eliasson and P. K. Shukla, Nonlinear quantum fluid equations for a finite temperature Fermi plasma, Phys. Scr. 78, 025503 (2008). doi:10.1088/0031-8949/78/02/025503 